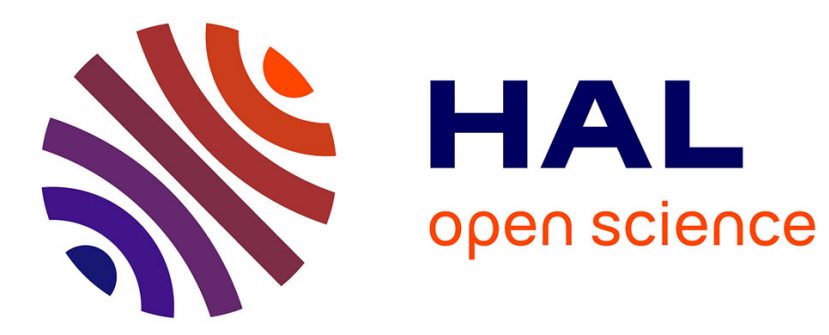

\title{
Stochastic Modeling of Impulse Responses in Reverberating Environments
}

Andrea Cozza

\section{To cite this version:}

Andrea Cozza. Stochastic Modeling of Impulse Responses in Reverberating Environments. IEEE Transactions on Vehicular Technology, 2018, 10.1109/TVT.2018.2876296 . hal-01825315v2

\section{HAL Id: hal-01825315 \\ https://hal.science/hal-01825315v2}

Submitted on 13 Oct 2018

HAL is a multi-disciplinary open access archive for the deposit and dissemination of scientific research documents, whether they are published or not. The documents may come from teaching and research institutions in France or abroad, or from public or private research centers.
L'archive ouverte pluridisciplinaire HAL, est destinée au dépôt et à la diffusion de documents scientifiques de niveau recherche, publiés ou non, émanant des établissements d'enseignement et de recherche français ou étrangers, des laboratoires publics ou privés. 


\title{
Stochastic Modeling of Impulse Responses in Reverberating Environments
}

\author{
Andrea Cozza
}

\begin{abstract}
Propagation of waves within media supporting reverberation is usually regarded as a direct extension of the case of multi-path propagation, where a set of independent paths, equivalent to plane-wave contributions, can be drawn between a transmitter and a receiver. This paper adopts an alternative approach, based on modal theory, in order to derive models of the stochastic behavior of impulse responses (IRs) measured within such media. IRs can be represented as stationary Gaussian random processes whose amplitude is modulated by a decay function that converges to an exponential only if the time constants of each mode are similar, otherwise displaying a decay rate slowing with time as the modal time constants become more diverse. The asymptotic convergence to a Gaussian process is controlled by the number of available modes, which modal theory predicts to increase linearly with the bandwidth, but quadratically with the frequency. Modal theory implies that groups of typically more than eight propagation paths must be coherently related in order to give rise to reverberation. As a result, fewer degrees of freedom may be available than expected from the number of propagation paths involved, thus leading to a slower convergence to Gaussian propagation models. The stochastic model introduced is further applied in order to understand how far IRs can locally fluctuate away from their root-mean-square amplitude profiles. All theoretical predictions are supported by experimental results.
\end{abstract}

Index Terms-Multipath propagation, modal theory, reverberation, indoor propagation, statistical electromagnetics, random processes, time-domain analysis, impulse responses.

\section{INTRODUCTION}

Partially or fully closed environments often provide the setting where waves evolve, in particular for wireless communications. Examples are indoor and dense urban environments, hangars, industrial plants, mines, in-vehicle communications, etc. All of them share a common feature, namely the fact that waves undergo a large number of scattering events over reflective boundaries (e.g., walls), leading to radiated energy being distributed along multiple directions, thus generating multiple propagation paths between a transmitter and a receiver. Depending on the geometry of the environment, and the relative positions of transmitters and receivers, the timeof-flight of each separate path may vary significantly, giving rise to time-spread versions of originally transmitted signals [1], [2].

Impulse responses (IRs) in reverberant media display two separate contributions: a) deterministic contributions related

Andrea Cozza is with GeePs, Group of electrical engineering - Paris UMR CNRS 8507, CentraleSupelec, Univ Paris-Sud, Sorbonne Universités, UPMC Univ Paris 06, 11 rue Joliot Curie, Plateau de Moulon F91192 Gif sur Yvette CEDEX.

This work was partially supported by the French National Research Agency through the grant ANR-12-ASTR-0005, MIMOCHIC project, within the framework of the 2012 ASTRID program.

Contact e-mail: andrea.cozza@supelec.fr to propagation paths subject to a few specular reflections, observed in the early-time response and b) random contributions resulting from recursive multiple reflections and scattering events, usually referred to as a diffuse field or tail, appearing later in time. These two groups require different modeling approaches, as highlighted in [3] and references therein. This paper will not address deterministic contributions, rather focusing on diffuse multi-path contributions, whose macroscopic features are usually captured by means of shape factors, such as their power-delay profile (PDP), i.e., the time-dependent average instantaneous power of IRs observed through a medium.

A large variety of PDP models is available in the literature. A classical example is Saleh-Valenzuela multiple-cluster model [4], which can describe the general case of clusters of delayed contributions, each decaying in time according to an exponential function. PDPs can then be thought of as modulating random processes that describe the noise-like behavior of individual IRs. These random processes have been found to broadly follow a Gaussian distribution in many cases [5]-[8].

The main handicap of this kind of macroscopic models is their inability to provide physical insight into the conditions that ensure their validity. But the fact that exponential decays be systematically observed in certain practical settings, when involving time constants larger than the time-of-flight delay needed to cross the environment, is a direct hint at their ability to support reverberation [9], [10]. A reverberant response enables the use of modal descriptions, as the one proposed in this paper, which is here shown to formally prove that an exponential decay can, under certain conditions, be a good approximation for PDPs.

Moreover, the approximate Gaussian behavior of IRs has mostly been based on empirical data or by adopting asymptotic descriptions such as Rayleigh or Rice diffusion. These two asymptotic models are often invoked as soon as a propagation medium is expected to support a large number of propagation paths which, once assumed to be statistically independent, naturally lead to diffusive conditions, implying that IRs can be described as Gaussian random processes. The problem is, the conditions under which these approximations hold are seldom discussed, with no quantitative approach to stochastic convergence, apart assuming the availability of a large number of degrees of freedom (DoF).

The first aim of this paper is to provide a formal framework to model the stochastic behavior of IRs based on physical grounds, acknowledging from the beginning the peculiarities of reverberant environments. The proposed models are derived using modal theory, which fits practical observations in case of 


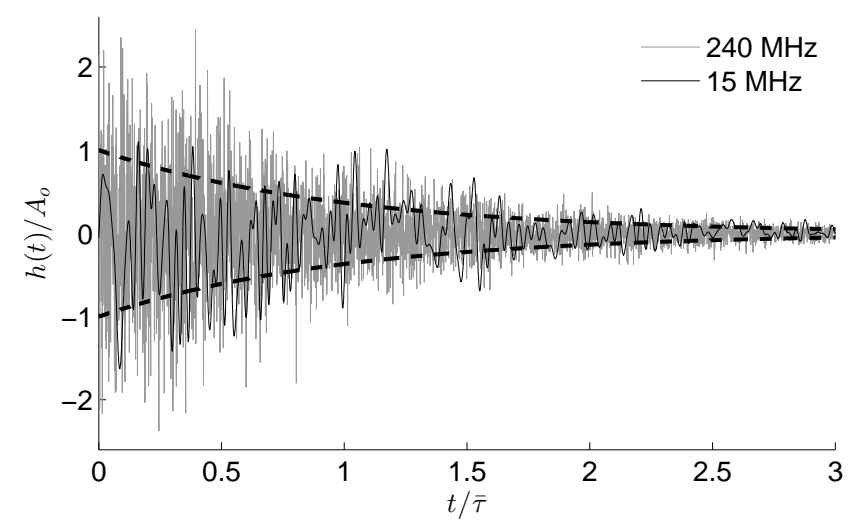

Figure 1: Two IRs measured at $2 \mathrm{GHz}$ in a reverberation chamber (see Sec. VII), for two different bandwidths. Notice the marked difference in the fluctuations dynamics even though the two IRs share the same root-mean-square (rms) envelope (marked as dashed lines).

reverberant environments. Its main appeal is physical insight and the ability to yield quantitative predictions from a handful parameters, such as the volume of the propagation medium and the frequency range over which it is used.

Our derivations focus on the case of a single-cluster PDP, which comes with a mathematical description simple enough as to yield insightful understanding of the physical phenomena behind their complex behavior. The description introduced in this paper can be extended to more general cases, in order to account for multiple clusters, but would make physical interpretation less straightforward. Moreover, multiple references suggest that PDPs observed in reverberating environments are mostly characterized by a single decaying exponential in indoor environments [5], [10], urban settings [7], [11], commercial airplanes [12], [13], at the interior of vehicles [14][18], industrial sites [8], [19], as well in metallic ducts [20]. Finally, laboratory facilities emulating wireless communications in realistic media are based on the use of reverberation chambers [17], which typically display only a single cluster of delayed power contributions.

The proposed model of IRs as non-stationary random processes is subsequently used in order to predict how strongly individual IRs sharing the same PDP can differ on a local scale; Fig. 1 illustrates this issue for two IRs measured within the same medium. Several statistical metrics are used to this effect, such as their peak instantaneous power and the probability of occurrence of undetectable contributions. All theoretical predictions are fully supported by experimental data measured in a reverberation chamber.

The paper starts by arguing on the fundamental differences between multi-path and modal descriptions in Sec. II, and in particular the number of DoF that could be expected in the two cases. A modal description is then adopted in Sec. III, proving that IRs in reverberating media behave as non-stationary random processes that can be asymptotically factorized as the product of a Gaussian stationary process and the IRs envelope, which can be approximated by a decaying exponential. These results are further developed in Secs. IV to VI, where local or instantaneous fluctuations in random realizations of IRs are studied. Sec. VII presents a thorough experimental validation of these models and the related predictions, in particular that the number of modes appear to be the dominant factor behind the convergence to a Gaussian random process. Most graphs issued from theoretical models are only shown in Sec. VII, where they serve as theoretical references for experimental results.

\section{Propagation models And expected DoF}

Transfer functions in complex media can be described by means of tap delay-line models [4], [7], [21], [22]

$$
H(\omega)=\sum_{n=1}^{N} a_{n} \mathrm{e}^{-\mathrm{j} \omega \tau_{n}},
$$

with $\tau_{n}$ the delay associated to the $n$th contribution, $a_{n}$ its complex amplitude and $\omega$ the angular frequency. Depending on the way these parameters are chosen [23], in particular their eventual frequency dependence, (1) can represent a series of distinct echoes, a continuous spread, i.e., reverberation, or a series of clustered contributions. In all of these cases, each contribution in (1) is interpreted as a distinct path along which a portion of the transmitted energy propagates. In case these multiple paths were different enough to be regarded as statistically independent, asymptotic models such as those for Rice and Rayleigh channels could be invoked. More realistic models can be considered, where groups of paths are partially correlated together, while intergroup correlation is regarded as negligible, but in practice are not used due to impossibility of predicting or measuring partial correlation.

When each individual contribution to (1) is interpreted as a (locally) plane wave propagating along a given path [24], the position $\boldsymbol{r}$ of the receiver/observer can be introduced in (1)

$$
H(\omega ; \boldsymbol{r})=\hat{\boldsymbol{p}}_{r} \cdot \sum_{n=1}^{N} \hat{\boldsymbol{p}}_{n} b_{n}(\omega) \mathrm{e}^{-\mathrm{j} \boldsymbol{k}_{n} \cdot \boldsymbol{r}}
$$

where $\hat{\boldsymbol{p}}_{r}$ is the polarization of the receiver, $\boldsymbol{k}_{n}=k_{o} \hat{\boldsymbol{k}}_{n}$ gives the direction along which the $n$th plane wave approaches the receiver, with a complex weight $b_{n}(\omega)$, and a polarization $\hat{\boldsymbol{p}}_{n}$; $k_{o}=\omega / c_{o}$ is the wave-number for a speed of light $c_{o}$. The receiver is assumed to be isotropic, for the sake of simplicity.

Although this family of models is completely general, it comes with an unanswered question: should $N$ be regarded as a measure of the number of DoF available for transmitting signals? In order to answer this question, one should assess how independent are these $N$ contributions, but they are usually regarded as independent, to the best of our knowledge; e.g., [25] considers a ray-tracing propagation model assuming each path to be independent.

Such an assumption is reasonable for configurations where scatterers such as walls and buildings act as guiding structures, i.e., where energy propagates about a main direction. But these assumptions break down when dealing with closed environments, where waves may propagate back and forth several times, giving rise to standing-wave patterns that underly diffusion and reverberation. In this case subgroups of paths must 

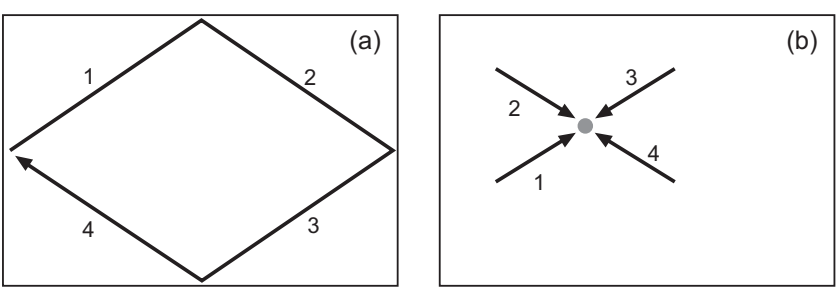

Figure 2: Modal versus plane-wave representations in a closed environment: (a) plane-wave contributions in a periodic-orbit mode (standing wave), generated and self-sustained sequentially leading to a global coherent pattern and (b) the subjective perception of an observer (gray spot), experiencing on a local level what appear to be four independently impinging plane waves.

be organized in closed coherent patterns [26, Ch. 3], especially in presence of planar surfaces, a signature of integrable wave billiards [27]. This physical fact is important since it implies that groups of waves impinging on the receiver are not acting as separate entities. These ideas are schematically illustrated in Fig. 2. As a result, the number of propagation paths should not be regarded as a measure of the DoF available in these media; one should rather consider the number of patterns, or modes, allowed by the geometry and materials involved in an environment.

There is no simple way of assessing beforehand the relationship between the number of resonant modes and of propagation paths, apart for very simple geometries. Typically, the number of paths ranges one order of magnitude above the number of standing-wave patterns. It is thus clear that regarding paths as DoF may lead to overestimate the probability of experiencing asymptotic conditions (e.g., diffusion) in reverberating media.

The average number of modes supported by a closed structure obeys a universal law requiring little prior information, first derived by $\mathrm{H}$. Weyl, which relates space to frequency, by exploiting the fact that for a given volume only certain patterns (or modes) can self-sustain, and only around a few selected frequencies. As reported in [28],

$$
M \simeq \frac{8 \pi}{c_{o}^{3}} V f_{c}^{2} B_{T}=8 \pi V_{\lambda} \frac{B_{T}}{f_{c}},
$$

where $M$ is the average number of modes resonating within a bandwidth $B_{T}$ centered at the frequency $f_{c}$, for a structure of volume $V ; \lambda$ is the wavelength associated to $f_{c}$, for a speed of light $c_{o}$, while $V_{\lambda}$ stands for the volume expressed in cubic wavelengths. Eq. (3) holds as long as $B_{T}$ is greater than the coherence bandwidth $B_{c}$ of the medium, otherwise $M$ should rather be estimated as the number of modes overlapping at $f_{c}$ [29], [30], a case not considered in this paper.

The modal-theory alternative to (2) is a discrete sum of modes $\boldsymbol{e}_{k}(\boldsymbol{r})$ [31, Sec. 13.1] [32, Ch. 10], proper to the medium's geometry and boundary conditions

$$
H(\omega ; \boldsymbol{r})=\hat{\boldsymbol{p}}_{r} \cdot \sum_{k=1}^{M} \gamma_{k} \boldsymbol{e}_{k}(\boldsymbol{r}) \psi_{k}(\omega),
$$

where $\gamma_{k}$ is the modal weight for the $k$ th mode resonating at $\omega_{k}$ with a quality factor $Q_{k}$, according to the frequency response

$$
\psi_{k}(\omega)=\left[\omega^{2}-\omega_{k}^{2}\left(1-\mathrm{j} / Q_{k}\right)\right]^{-1}
$$

The $\left\{\gamma_{k}\right\}$ are a function of the source of radiation, but are given once its nature and position are fixed. In fact, their actual value is not important when adopting a stochastic framework, as discussed later.

Eqs. (1) and (4) are mutually compatible since, as intuitively suggested in Fig. 2(a), each modal distribution $\boldsymbol{e}_{k}(\boldsymbol{r})$ can be expanded into $N_{k}$ plane waves, i.e.,

$$
H(\omega ; \boldsymbol{r})=\hat{\boldsymbol{p}}_{r} \cdot \sum_{k=1}^{M} \gamma_{k} \psi_{k}(\omega) \sum_{p=1}^{N_{k}} b_{k p} \mathrm{e}^{-\mathrm{j} \boldsymbol{k}_{k p} \cdot \boldsymbol{r}} .
$$

The main difference is that modal theory states that the planewave coefficients $\left\{b_{k p}\right\}$ only depend on the environment and not on the source/transmitter and the receiver characteristics. Therefore, even though $\sum_{k=1}^{M} N_{k}$ plane waves are propagating, only the $\left\{\gamma_{k}\right\}$ can modify the way these plane waves are excited, and this only through sub-groups pertaining to each mode.

The perception of the number of available DoF is therefore strongly affected. In particular, from (3) is can be expected that increasing $f_{c}$ should lead to a faster convergence to Rayleigh diffusion than a comparable increase in the bandwidth $B_{T}$, a prediction that cannot be derived from a classical multi-path model as (2). This prediction is put to test in Sec. VII.

\section{MODAL-BASED MODEL FOR $h(t)$}

Inverse transforming (5), the time-domain response of a single mode resonating at the angular frequency $\omega_{k}$ can be written as

$$
\psi_{k}(t)=\mathrm{e}^{-t / \tau_{k}} \sin \left(\omega_{k} t\right) u(t)
$$

with $\tau_{k}=2 Q_{k} / \omega_{k}$ the time constant related to the quality factor $Q_{k}$ of the resonance and $u(t)$ Heaviside step function. Introducing the coefficients $\left\{a_{k}\right\}$

$$
a_{k}=\gamma_{k} \hat{\boldsymbol{p}}_{r} \cdot \boldsymbol{e}_{k}(\boldsymbol{r})=\alpha_{k} \mathrm{e}^{\mathrm{j} \varphi_{k}}
$$

(4) can be written as

$$
h(t)=u(t) \sum_{k=1}^{M} \mathrm{e}^{-t / \tau_{k}} \alpha_{k} \sin \left(\omega_{k} t+\varphi_{k}\right),
$$

where the observer's position has been dropped for the sake of brevity.

The $\left\{\gamma_{k}\right\}$ can typically be assumed to be independent and identically distributed [33], in particular for the case where no line-of-sight (LoS) contribution is present, as assumed in all results and discussions reported in the rest of this paper. This same property is inherited by the $\left\{a_{k}\right\}$. More general scenarios can be reinstated by adding a LoS contribution to a purely non-LoS case, as done, e.g., in [7], a case not treated in this paper.

As a result of these assumptions, all the sine functions display independent random phase-shift angles, with $\left\{\varphi_{k}\right\} \sim$ $\mathscr{U}(0,2 \pi)$, i.e., uniformly distributed over all possible angles. 
Moreover, the random nature of the frequencies $\left\{\omega_{k}\right\}$ also act as sources of randomness in the phase terms [27]. It can therefore be shown that under these assumptions $\langle h(t)\rangle=0, \forall t$, where $\langle\cdot\rangle$ stands for the ensemble average of a random process or variable.

The IR model here sought is given by the product of the square root of the PDP, i.e., the IR envelope $h_{o}(t)$, and a zeroaverage random process, $w(t)$,

$$
h(t)=h_{o}(t) w(t),
$$

where the IR envelope $h_{o}(t)$ is expected to take the shape

$$
h_{o}(t)=A_{o} \mathrm{e}^{-t / \tau} u(t),
$$

where $\tau$ is a time constant typically associated to dissipation/leakage phenomena within a propagation environment. An exponential-decay law can be derived on the basis of a first-order dissipation model, for very narrow-band conditions under Rayleigh diffusion [34], [35]. This property is here demonstrated to be just a special case of our model, derived under more general conditions.

From (9), the PDP of $h(t)$ is found as

$$
\begin{aligned}
& h_{o}^{2}(t)=\left\langle h^{2}(t)\right\rangle=\left\langle\sum_{k=1}^{M} \mathrm{e}^{-2 t / \tau_{k}} \alpha_{k}^{2} \sin ^{2}\left(\omega_{k} t+\varphi_{k}\right)\right\rangle \\
& +\left\langle\sum_{p \neq q} \mathrm{e}^{-2 t / \tau_{p}} \mathrm{e}^{-2 t / \tau_{q}} \alpha_{p} \alpha_{q} \sin \left(\omega_{p} t+\varphi_{p}\right) \sin \left(\omega_{q} t+\varphi_{q}\right)\right\rangle .
\end{aligned}
$$

Because of the assumptions on the modal weights $\left\{\gamma_{k}\right\}$, the second average in (12) is vanishing with respect to the first one. Assuming a weak dependence between the sets $\left\{\gamma_{k}\right\}$, $\left\{\omega_{k}\right\}$ and $\left\{\tau_{k}\right\}$ and invariant probability laws with respect to $k$

$$
h_{o}^{2}(t)=\left\langle\mathrm{e}^{-2 t / \tau_{k}}\right\rangle\left\langle\alpha_{k}^{2}\right\rangle M / 2 .
$$

The first average only involves the modal time constants

$$
\left\langle\mathrm{e}^{-2 t / \tau_{k}}\right\rangle=\int \mathrm{d} \tau p_{\tau}(x) \mathrm{e}^{-2 t / x},
$$

where $p_{\tau}(x)$ is the probability density function of $\tau_{k}$. For the sake of simplicity, the $\tau_{k}$ are here assumed to be uniformly distributed around their average $\bar{\tau}$, covering a total span $2 \Delta \tau$, for which

$$
\left\langle\mathrm{e}^{-2 t / \tau_{k}}\right\rangle=(2 c)^{-1} \int_{-c}^{c} \mathrm{~d} s \mathrm{e}^{-2 t / \bar{\tau}(1+s)},
$$

with $c=\Delta \tau / \bar{\tau}$. Relevant discussions about the behavior of $\left\{Q_{k}\right\}$, and therefore $\left\{\tau_{k}\right\}$ can be found in [36], [37]. While these results would differ when choosing other distributions, the main goal of (15) is to assess how sensitive is the drift from a pure exponential decay in case of diverse $\left\{\tau_{k}\right\}$.

Solving (15) yields

$$
\left\langle\mathrm{e}^{-2 t / \tau}\right\rangle=\mathrm{e}^{-2 t / \bar{\tau}} \eta^{2}(t, c),
$$

where $\eta(t, c)$ is given by

$$
\eta^{2}(t, c)=g(t, c)-g(t,-c)
$$

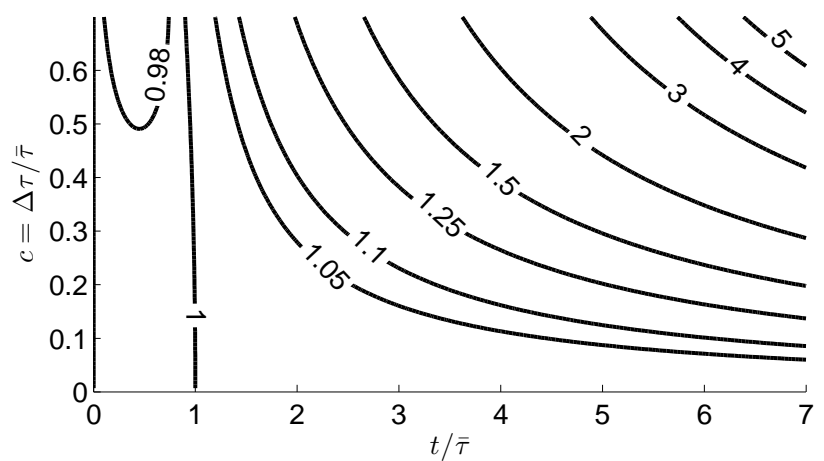

Figure 3: Contour plot of the drift function $\eta(t, c)$.

and

$$
g(t, s)=\frac{1+s}{2|s|} \exp \left(\frac{2 t}{\bar{\tau}} \frac{s}{1+s}\right)+\frac{t / \bar{\tau}}{|s|} \mathrm{e}^{2 t / \bar{\tau}} \operatorname{Ei}\left(-\frac{2 t / \bar{\tau}}{1+s}\right)
$$

with $\operatorname{Ei}(\cdot)$ the exponential integral. $\eta(t, c)$ measures how much the IR envelope $h_{o}(t)$ differs from the pure exponential decay appearing on the right-hand side of (16). For $t / \bar{\tau} \gg 1, \eta(t, c)$ can be approximated as

$\eta^{2}(t, c)=\frac{(c+1)^{2}}{4 c t / \bar{\tau}} \exp \left[\frac{2 c}{c+1} \frac{t}{\bar{\tau}}\right]-\frac{(c-1)^{2}}{4 c t / \bar{\tau}} \exp \left[\frac{2 c}{c-1} \frac{t}{\bar{\tau}}\right]$,

proving an exponential drift of the late-time PDP from the purely exponential decay $\exp (-2 t / \bar{\tau})$, as soon as $c>0$. For this reason $\eta(t, c)$ will be referred to as the drift function.

Summarizing the above results, (13) yields

$$
h_{o}(t)=A_{o} \mathrm{e}^{-t / \bar{\tau}} \eta(t, c) u(t),
$$

with

$$
A_{o}=m_{\alpha} \sqrt{M / 2}
$$

and $m_{\alpha}^{2}=\left\langle\alpha_{k}^{2}\right\rangle$. Eq. (20) confirms the validity of an exponential envelope, as postulated in (11), only if $\eta(t, c) \simeq 1, \forall t, c$. The drift function is mapped in Fig. 3, where it appears that $\eta(t, c)$ is not necessarily close to one. For $t<\bar{\tau}$ a shallow region finds $\eta(t, c) \simeq 1$, but for later instants $c$ should not be larger than $20 \%$ for this condition to hold. Stated in other terms, an exponential envelope should not be taken for granted and is in itself an indirect proof of weak dispersion in the distribution of the random $\left\{\tau_{k}\right\}$. The prediction of a drift function $\eta(t, c) \geq 1$ is confirmed in Sec. VII.

The squared envelope $h_{o}^{2}(t)$ is meant to describe the way energy evolves (on average) in the response of a reverberating medium when driven by an excitation pulse. While this kind of model is useful in order to characterize the power-delay spread of a channel, it does not provide detailed information about the potential differences in the IRs sharing the same power-delay profile.

More insight can be obtained by analyzing the statistical properties of $w(t)=h(t) / h_{o}(t)$. From (9), (13) and (21)

$$
w(t)=\sqrt{2 / M} \sum_{k=1}^{M} \beta_{k} \sin \left(\omega_{k} t+\varphi_{n}\right)
$$


where $\beta_{k}=\alpha_{k} / m_{\alpha}$. The statistical properties of $w(t)$ can be derived by noticing that the summation in (22) can be interpreted as a $2 \mathrm{D}$ random walk, since

$$
\sum_{k=1}^{M} \beta_{k} \sin \left(\omega_{k} t+\varphi_{k}\right)=\operatorname{Im}\{s(t)\},
$$

where

$$
s(t)=\sum_{k=1}^{M} \beta_{k} \mathrm{e}^{j \xi_{k}}
$$

having set $\xi_{k}=\omega_{k} t+\varphi_{k}$. Since $\left\{\varphi_{k}\right\} \sim \mathscr{U}(0,2 \pi)$, i.e., uniformly distributed, also $\left\{\xi_{k}\right\} \sim \mathscr{U}(0,2 \pi), \forall t, \omega_{k}$. The summation in (23) can now be recognized as a random walk in the complex plane, with random steps along random directions $\left\{\xi_{k}\right\}$. This class of random processes is known to present a probability distribution with circular symmetry, i.e., only dependent on the norm of $s(t)$. The central-limit theorem iimplies that $s(t)$ can be approximated as a Gaussian process; hence, $s(t)$ is fully characterized by its first two moments. In practice, only the variance $\sigma_{s}^{2}$ of $s(t)$ needs to be computed, as the isotropy of $s(t)$ leads straightforwardly to an average equal to zero. It can be shown that $\sigma_{s}^{2}=M, \forall t$, i.e., $s(t)$ is stationary, while the isotropy of $s(t)$ results into $\left\langle\operatorname{Im}\{s(t)\}^{2}\right\rangle=\left\langle|s(t)|^{2}\right\rangle / 2$. Convergence to this kind of process is consistent with observations in real-life scenarios, as presented, e.g., in [8], [12], [22] and indirectly in [11], where a zero excess kurtosis was observed. A uniform phase-shift angle distribution was confirmed in [6], [38].

Taking into account these properties in (22), the first two moments of $w(t)$ become

$$
\begin{aligned}
\langle w(t)\rangle & =0 \\
\left\langle w^{2}(t)\right\rangle & =1
\end{aligned}
$$

$\forall t \geq 0$, i.e., $w(t)$ is a stationary process and follows a standard normal probability law. Since these conclusions are based on the central-limit theorem, they can be expected to hold as long as $M \gg 1$, while the individual contributions for each mode/resonance needs to be weakly correlated.

The modal description from which (22) was derived implies that the $M$ modes available act as independent degrees of freedom that will eventually have $w(t)$ converging to a Gaussian process. $M$ should therefore be expected to drive this convergence. Experiments presented in Sec. VII-C support this prediction and prove that even for $M \gg 1$ local deviations from Gaussianity are observed.

\section{PEAK-VALUE ATTAINED BY AN IR}

The factorization of $h(t)$ as in (10) makes it possible to assess how strongly single realizations of $h(t)$ can deviate from their envelope $h_{o}(t)$, on a local scale. Several metrics are considered in the following sections, starting with the probability distribution of the peak value $M_{h}=\max _{t}|h(t)| / A_{o}$. This is of practical importance, e.g., in assessing the probability of observing overshooting events, leading to increased peak-toaverage power ratios [15], but also for electromagnetic dosimetry in cavities [39] and assess the intensity of electromagnetic stress in EMC tests.
The IR $h(t)$ is first approximated as a discrete time-series, by sampling it at $t=n T_{c}$, with $T_{c}$ the coherence time discussed later in this section. The samples $\left\{h_{n}=h\left(n T_{c}\right)\right\}$ are required to be independent; being modeled as Gaussian random variables, this condition is equivalent to requiring uncorrelated data. The probability law of $M_{h}$ can now be expressed as

$$
F_{M_{h}}(x)=\mathrm{P}\left(M_{h}<x\right)=\mathrm{P}\left(\left|h_{n}\right|<x, \forall n\right),
$$

which, for independent $\left\{h_{n}\right\}$, translates into

$$
\mathrm{P}\left(M_{h}<x\right)=\prod_{n=0}^{\infty} \mathrm{P}\left(\left|h_{n}\right|<x\right),
$$

as each $\left|h_{n}\right|<x$ at the same time, i.e., representing $\mathrm{P}\left(M_{h}<x\right)$ as a joint probability. This kind of probability law is reminiscent of an extreme-value law [40], where the maximum value observed in a set of $N$ iid random variables is considered. The fundamental difference here is that the samples are not identically distributed, because of the decaying envelope $h_{o}(t)$.

Since $w(t)$ is asymptotically a Gaussian process, (10) has the $\left\{h_{n}\right\}$ also behaving as Gaussian random variables, with standard deviations $\sigma_{n} \simeq \exp \left(-n T_{c} / \bar{\tau}\right)$. The drift function $\eta(t, c)$ is here neglected since the peak-value of $|h(t)|$ is likely to occur for $t / \bar{\tau}<2$, where $\eta(t, c) \simeq 1$.

For a Gaussian $h_{n}$, its modulus $\left|h_{n}\right|$ follows by definition a half-normal distribution. Therefore, $\mathrm{P}\left(\left|h_{n}\right|<x\right)=$ $\left.\operatorname{erf}\left[\exp \left(n T_{c} / \bar{\tau}\right) / \sqrt{2}\right)\right]$, so that (27) results into

$$
F_{M_{h}}(x)=\prod_{n=0}^{\infty} \operatorname{erf}\left(x \mathrm{e}^{n T_{c} / \bar{\tau}} / \sqrt{2}\right),
$$

where erf is the error function.

As the standard deviation of late-time samples decreases exponentially, it is clear that their contribution to the peak value $M_{h}$ can be expected to be negligible. It is therefore possible to truncate the product in (29) to $K$ samples, where $K$ can be chosen such that the probability of having $\left|h_{n}\right|>A_{o}$ is negligible $\forall n>K$, e.g., lower than $5 \%^{1}$. This condition is met when the standard deviation of $h_{K} / A_{o}$ is smaller than $1 / 2$, i.e., $\exp \left(-K T_{c} / \bar{\tau}\right)<1 / 2$, hence

$$
K \leq\left(\bar{\tau} / T_{c}\right) \ln 2 .
$$

As a result, the only $t \leq K T_{c}$ is considered, i.e., $t / \bar{\tau} \leq \ln 2$, which falls in the region where $\eta(t, c) \simeq 1$, thus justifying the use of an exponential decay in the present derivation.

Fig. 4 shows a few examples of probability distribution for the overshoot factor $M_{h}$, obtained by numerically computing the derivative of (29) with respect to $x$. These results indicate that an IR can exceed by non-negligible margin its rms envelope $h_{o}(t)$ as $\bar{\tau} / T_{c}$ increases.

A few quantiles of (29) are shown in Fig. 5 versus $T_{c} / \bar{\tau}$. The mode of (29) as a function of $T_{c} / \bar{\tau}$, truncated according

\footnotetext{
${ }^{1}$ This probability should not be confused with that of having late-time samples producing the highest peak value, which is exceedingly smaller, because of the exponential decay
} 


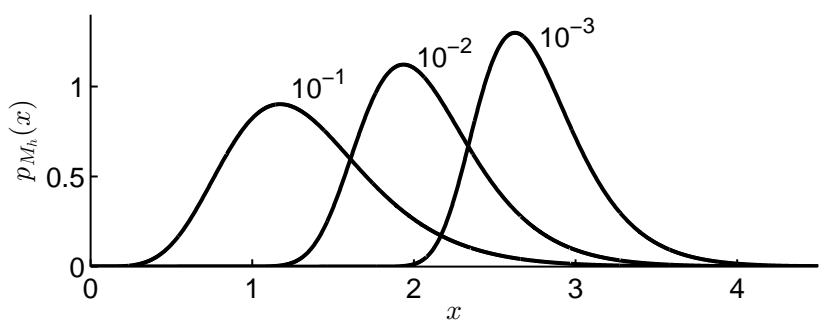

Figure 4: Probability density functions of $M_{h}=$ $\max _{t}|h(t)| / A_{o}$, as predicted by (29). Three values of $T_{c} / \bar{\tau}$ are considered, as displayed on top of each curve.

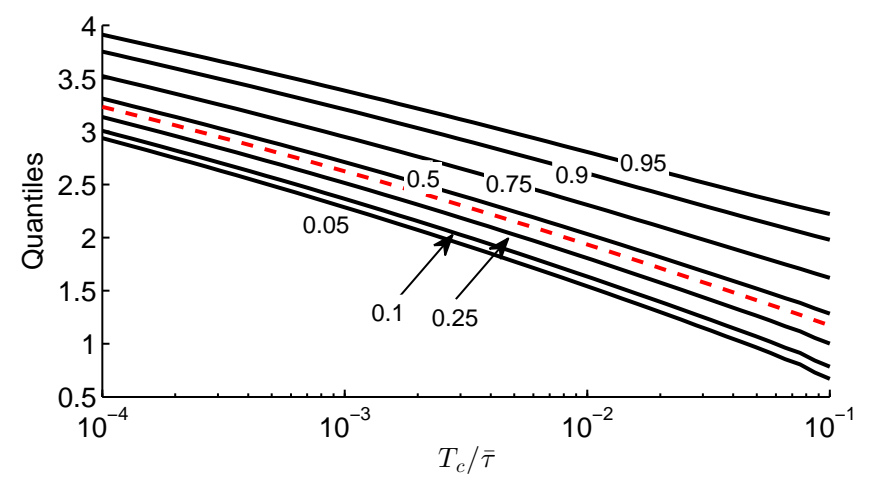

Figure 5: Quantiles of $M_{h}$ as functions of $T_{c} / \bar{\tau}$, with their associated probabilities superimposed over each curve. The red dashed curve represents the mode of $M_{h}$.

to (30), can be approximated, within an error of less than a percent point, as

$$
m\left(T_{c} / \bar{\tau}\right) \simeq a \ln ^{b}\left(\bar{\tau} / T_{c}\right)
$$

with $a=0.626$ and $b=0.741$ obtained by least-square fitting. In the same way, (31) approximates the average when $a=$ 0.749 and $b=0.678$.

The condition needed for independent $\left\{h_{n}\right\}$, i.e., uncorrelated $\left\{w_{n}\right\}$, can be inferred from the auto-correlation function of $w(t)$

$$
R_{w}(\tau)=\langle w(t) w(t+\tau)\rangle
$$

which, following (22), can be shown to be

$$
R_{w}(\tau)=\left\langle\cos \left(\omega_{n} \tau\right)\right\rangle
$$

Approximating the resonant frequencies $\left\{f_{n}\right\}=\left\{\omega_{n} / 2 \pi\right\}$ as uniformly distributed over the excitation bandwidth $B_{T}$ centered on $f_{c}$

$$
R_{w}(\tau)=\cos \left(2 \pi f_{c} \tau\right) \operatorname{sinc}\left(\pi B_{T} \tau\right)
$$

which is consistent with the results presented in [11].

The coherence time can then be assessed as the equivalent time support of the time-coherence function $\mu_{w}(\tau)=$ $R_{w}(\tau) / R(0)$

$$
T_{c}=\int_{0}^{\infty} \mathrm{d} \tau \mu_{w}^{2}(\tau)
$$

which, thanks to Parseval and Wiener theorems, can be expressed as

$$
T_{c}=\left[2 \int_{B_{T}} \mathrm{~d} \omega S_{w}(\omega)\right]^{-2} \int_{B_{T}} \mathrm{~d} \omega S_{w}^{2}(\omega),
$$

where $S_{w}(\omega)$ is the power spectral density of $w(t)$. Fourier transforming (34),

$$
S_{w}(\omega)=1 / 2 B_{T}
$$

over the excitation bandwidth $B_{T}$ and zero outside it, from which

$$
T_{c}=1 / 4 B_{T}
$$

\section{DYNAMIC RANGE OF FLUCTUATIONS}

The fact that the peak value of $h(t)$ can significantly stray away from the IR envelope $h_{o}(t)$ does not come as a surprise, since typical IRs in closed media are characterized by wide fluctuations. While their Gaussian nature explains their intensity, it is interesting to understand the dynamical range spanned by $|h(t)|$, i.e., to measure how strongly the amplitude of $h(t)$ can swing between two close observations. This information would allow to understand whether IR locally exceeding their envelope should be expected to rapidly fluctuate to much lower amplitudes in a short time span, thus appearing as a sequence of rapid pulsed bursts rather than a continuous decaying tail.

We therefore focus on the ratio $Z=|h(t+\Delta t) / h(t)|=$ $|w(t+\Delta t) / w(t)| \exp (-\Delta t / \bar{\tau})$ and compute how it is distributed, assuming that the two random variables involved in it are weakly correlated, i.e., $\Delta t>T_{c}$. The probability density function of the ratio $R=|w(t+\Delta t) / w(t)|$ can be obtained as

$$
p_{R}(x)=\int_{0}^{\infty} \mathrm{d} s p_{|W|}(s) \frac{\partial}{\partial x} F_{|W|}(x s),
$$

where $F_{|W|}(x)$ is the cumulative distribution of the modulus of $w(t), \forall t$. Since $|w(t)|$ follows a half-normal distribution law, with unitary standard deviation,

$$
\begin{aligned}
p_{R}(x) & =\int_{0}^{\infty} \mathrm{d} s(-s) p_{|W|}(s) p_{|W|}(x s) \\
& =\frac{2}{\pi} \int_{o}^{\infty} \mathrm{d} s(-s) \mathrm{e}^{\left(1+x^{2}\right) s^{2} / 2} \\
& =\frac{2}{\pi} \frac{1}{1+x^{2}} \quad, \quad x>0
\end{aligned}
$$

from which

$$
p_{Z}(x)=\mathrm{e}^{\Delta t / \bar{\tau}} p_{R}\left(x \mathrm{e}^{\Delta t / \bar{\tau}}\right) .
$$

The distributions (40) and (41) correspond to Cauchy distributions, whose average and standard deviation are not defined, known to model random variables with large stochastic dispersion. For the sake of simplicity we will hereafter assume $\Delta t \ll \bar{\tau}$, leading to $Z \simeq R$.

Because of the strong stochastic dispersion of $Z$, confidence intervals are more useful metrics to quantify its dynamic range. The cumulative distribution associated to $p_{Z}(x)$ is

$$
F_{Z}(x)=\frac{2}{\pi} \tan ^{-1}(x)
$$


and the quantile $q(p)$ for a probability $p$ is

$$
q(p)=F_{Z}^{-1}(p)=\tan \frac{\pi}{2} p
$$

so that the confidence interval for a significance level $\alpha$ is $[q(\alpha / 2), q(1-\alpha / 2)]$. It can be shown that (43) yields $q(\alpha / 2) q(1-\alpha / 2)=1, \forall \alpha$, as illustrated in Fig. 12, in Sec. VII-C. This result means that the probability of observing ratios smaller than $1 / C$ is equal to that of having them larger than $C$, where $C>0$. In other words, small observations can be followed by very large ones in the IR, and viceversa, $\forall t>T_{c}$, while the envelope $h_{o}(t)$ represents the median of the IRs. These considerations are important in the case of receivers with finite sensitivity, as discussed in the next section.

\section{UNDETECTABLE CONTRIBUTIONS}

Let a receiver/probe have a finite sensitivity $s_{o}$, defined as a fraction of $A_{o}$. This value could be set, e.g., by the level of background noise below which contributions are regarded as lost. Therefore, only those contributions such that $|h(t)|>s_{o} A_{o}$ will be regarded as detected. Taking the PDP as an estimate of the received instantaneous power, this condition is typically translated into a condition for the receiver to collect information only over a time interval up to $T_{o}=-\bar{\tau} \ln s_{o}$. E.g., in [2] a $30 \mathrm{~dB}$ dynamic is reported, i.e., $T_{o} / \bar{\tau}=3.45$. But because of the strong dynamic of random fluctuations discussed in Sec. V, there is a risk of experiencing significant reduction in instantaneous power with respect to what could be expected from the PDP.

On the other hand, the existence of overshooting events, as discussed in Sec. IV, would make one think that the total received energy could be a strongly fluctuating function and might even be higher than that expected from the PDP.

In order to elucidate these questions, we compute the probability of receiving undetectable contributions, starting from (10),

$$
\begin{aligned}
P_{o}(t)= & \mathrm{P}\left(|h(t)|<A_{o} s_{o}\right)=\mathrm{P}\left(|w(t)|<\exp (t / \bar{\tau}) s_{o}\right) \\
& =\operatorname{erf}\left(\exp (t / \bar{\tau}) s_{o} / \sqrt{2}\right),
\end{aligned}
$$

having assumed $\eta(t, c) \simeq 1$ in order to obtain closed-form results. The probability of not receiving significant contributions at $T_{o}$ is $P_{o}\left(T_{o}\right)=\operatorname{erf}(1 / \sqrt{2}) \simeq 0.68$, and is independent from $s_{o}$. The results presented in Fig. 13 in Sec. VII-C show that there is a non-negligible probability of not receiving any detectable contribution even in early-time observations, depending on the value taken by $s_{O}$.

Eq. (44) allows estimating the fraction of time $L_{N}\left(s_{o}\right)$, over the interval $t<T_{o}$, during which no detectable contributions are received on average

$$
\begin{aligned}
L_{N}\left(s_{o}\right) & =\frac{1}{T_{o}} \int_{0}^{T_{o}} \mathrm{~d} t P_{o}(t) \\
& =\frac{1}{T_{o}} \int_{0}^{T_{o}} \mathrm{~d} t \operatorname{erf}\left(\mathrm{e}^{t / \bar{\tau}} s_{o} / \sqrt{2}\right) \\
& =\frac{1}{-\ln s_{o}} \int_{0}^{-\ln s_{o}} \mathrm{~d} y \operatorname{erf}\left(\mathrm{e}^{y} s_{o} / \sqrt{2}\right),
\end{aligned}
$$

which is well approximated by

$$
L_{N}\left(s_{o}\right) \simeq-\frac{17-18 s_{o}+s_{o}^{3}}{9 \sqrt{2 \pi} \ln s_{o}} \quad, \quad s_{o}<1,
$$

obtained using the third-order Taylor expansion of erf $(x)$ for $x \simeq 0$, before computing the integral. Results in Fig. 14 imply that even for small values of $s_{o}$ there is a high probability of receiving no contribution, and that IRs should be expected to be highly fragmented.

It could therefore be expected that the total detectable energy be significantly smaller than what the PDP indicates. This point can be clarified by computing the instantaneous power detected on average

$$
\left\langle P_{d}(t)\right\rangle=\left\langle|h(t)|^{2}|| h(t) \mid>s_{o} A_{o}\right\rangle
$$

conditioned to detectable contributions, which can be obtained as

$$
\begin{gathered}
\left\langle P_{d}(t)\right\rangle=\sqrt{\frac{2}{\pi}} h_{o}^{-1}(t) \int_{s_{o} A_{o}}^{\infty} \mathrm{d} h h^{2} \mathrm{e}^{-h^{2} / 2 h_{o}^{2}(t)} \\
=\sqrt{\frac{2}{\pi}} s_{o} h_{o}(t) \mathrm{e}^{-s_{o}^{2} / 2 h_{o}^{2}(t)}+h_{o}^{2}(t) \operatorname{erfc}\left(\frac{s_{o}}{\sqrt{2} h_{o}(t)}\right) .
\end{gathered}
$$

The fraction of energy detected on average with respect to the energy associated to the PDP is then defined as

$$
L_{E}\left(s_{o}\right)=\frac{1}{\mathscr{E}_{P D P}\left(s_{o}\right)} \int_{0}^{T_{o}} \mathrm{~d} t\left\langle P_{d}(t)\right\rangle
$$

with

$$
\mathscr{E}_{P D P}\left(s_{o}\right)=\int_{0}^{T_{o}} \mathrm{~d} t h_{o}^{2}(t)=\frac{A_{o}^{2} \bar{\tau}}{2}\left(1-s_{o}^{2}\right),
$$

yielding

$$
L_{E}\left(s_{o}\right)=\sqrt{\frac{2}{\pi}} \frac{s_{o} \mathrm{e}^{-s_{o}^{2} / 2}-s_{o}^{2} / \sqrt{\mathrm{e}}}{1-s_{o}^{2}}+\operatorname{erfc}\left(\frac{s_{o}}{\sqrt{2}}\right),
$$

which can be approximated as

$$
L_{E}\left(s_{o}\right) \simeq 1-s_{o}^{2} \sqrt{2 / \pi \mathrm{e}} \quad, \quad s_{o} \lesssim 1 .
$$

Fig. 14 in Sec. VII-C proves that $L_{E}\left(s_{o}\right)$ stays remarkably close to one, even when $s_{o}$ increases to relatively high values. This outcome implies that even though $h(t)$ is substantially affected by undetectable contributions, its overall energy is very weakly affected on average, which makes sense only if the detected contributions compensate for the missing energy from those gone undetected, i.e., by means of frequent overshooting events. This explanation is indeed consistent with the results in Sec. V, where it was shown that very weak contributions can be followed by large ones that have the potential to compensate for missing contributions.

\section{EXPERIMENTAL VALIDATION}

Previous theoretical results were validated against experimental data measured in a reverberation chamber (RC), according to the setup and protocol described in Sec. VII-A. This choice is motivated by their wide-spread use as standard test facilities capable of emulating a large number of signaltransmission settings for strongly multi-path environments 

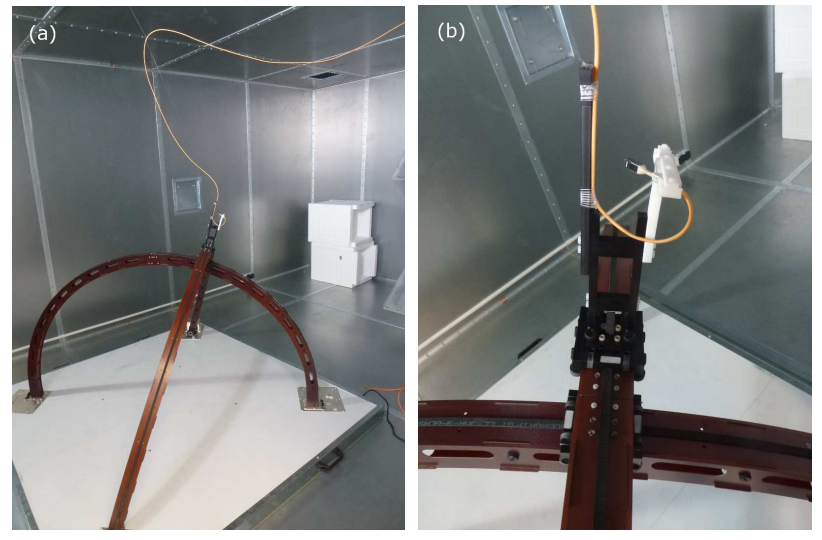

Figure 6: The hemispherical positioner (a) and the electrooptical probe (b) used in the experimental validation.

[16], [17], [41]. The fact that they present a similar statistical behavior even when taking different implementations (shape factors, volume, etc.) enables reproducible results.

Sec. VII-B first proceeds to the validation of the nonstationary random model introduced in Sec. III. Convergence to asymptotic results is shown to improve with the number of modes available, as argued in Sec. II. Substantial drifts from an exponential decay are found to be accurately described by the proposed model. Local deviations of $w(t)$ from a Gaussian distribution are highlighted. In spite of these deviations, results in Sec. VII-C prove that the asymptotic models derived in Secs. IV to VI for random fluctuations in IRs are remarkably accurate and can therefore be used as predictive tools.

\section{A. Test setup}

The experiments were carried out in a metallic RC, a cuboid of about $6 \times 3.5 \times 2.5 \mathrm{~m}^{3}$. The $\mathrm{RC}$ was equipped with a Zfolded metallic stirrer which, though not operated during the experiments, was fundamental for breaking symmetries within the RC.

A monocone antenna, impedance matched to ensure a return loss higher than $15 \mathrm{~dB}$ between 0.4 and $3 \mathrm{GHz}$ acted as transmitter. It was placed behind the stirrer, in order to minimize line-of-sight contributions in received signals. These were measured over a large number of positions, thanks to the hemispherical scanner shown in Fig. 6(a). This robot has the ability to ensure a very low level of perturbation at frequencies below $3 \mathrm{GHz}$, with a level of coherence higher than $70 \%$ when one of its arms moves [42]. The received signals were obtained by means of an EFS-105 probe manufactured by Enprobe, a linearly polarized electro-optical probe with flat wide-band receiving characteristics, shown in Fig. 6(b). Data were collected around two frequencies, at 0.5 and $2 \mathrm{GHz}$, for which (3) predicts a 16-fold increase in the number of DoF, for a given bandwidth $B_{T}$.

For each position of the receiving probe the transfer function between the monocone antenna and the probe was measured using a vector network analyzer from Rohde \& Schwarz, model ZVB8, acquiring 5000 frequency samples uniformly distributed over a $250 \mathrm{MHz}$ bandwidth, around 0.5 and $2 \mathrm{GHz}$.
The frequency step was chosen in order to limit the inevitable effects of time-domain aliasing, since the time constant of the $\mathrm{RC}$ was expected in the range of microseconds.

To make sure that the transfer functions were independent, the probe positions were chosen to be at least a quarter of wavelength away from each other [43], and uniformly distributed over the hemispherical surface allowed by the scanner. This resulted in a total of 244 positions at $0.5 \mathrm{GHz}$ and 888 at $2 \mathrm{GHz}$.

IRs were then retrieved by means of inverse discrete Fourier transforms, by having first applied a tapering function to the frequency-domain data. Tchebychev window is an effective choice to reduce the level of side lobes, with a time-domain counterpart $p(t)$, whose sampled representation is [44]

$$
p(k)=\frac{\cos \left(N \cos ^{-1}(\beta \cos \pi k / N)\right)}{\cosh \left(N \cosh ^{-1} \beta\right)},
$$

with $\beta=\cosh \left(N^{-1} \cosh ^{-1} 10^{\alpha}\right)$, and $N$ the number of frequency samples spanning the bandwidth $B_{T}$ of the IR of interest. In order for the late-time region of the IRs to be untainted by $p(t)$ over at least $60 \mathrm{~dB}$ of dynamics, we set $\alpha=4$, in order to ensure a side-lobe level below $-80 \mathrm{~dB}$. Moreover, for $p(t)$ to have a support way smaller than $\bar{\tau}$, $B_{T} \gtrsim 10 \mathrm{MHz}$, in order not to alter the early-time region of the IRs.

\section{B. Modal description and convergence}

The rms envelope $h_{o}(t)$ was first estimated from experimental data, as defined in (12), and $w(t)=h(t) / h_{o}(t)$ was then tested for Gaussianity and stationarity.

According to Fig. 3, $h_{o}(t)$ can be expected to be well approximated by an exponential decay only for $t / \bar{\tau}<2$, an interval covering the first $17 \mathrm{~dB}$ in $h_{o}(t)$, where $\eta(t, c) \simeq 1$ even for large dispersions of the modal $\left\{\tau_{k}\right\}$. The two parameters $A_{o}$ and $\bar{\tau}$ can then be retrieved by means of a least-square regression on this portion of data, leading to the dashed line in Fig. 7(a), obtained for $B_{T}=20 \mathrm{MHz}$. The same $\bar{\tau}=2.17 \mu \mathrm{s}$ was found for 0.5 and $2 \mathrm{GHz}$. Higher fluctuations for $0.5 \mathrm{GHz}$ are due to the more limited number of IRs available at that frequency.

Fig. 7(a) confirms that $h_{o}(t)$ drifts away from an exponential function for $t / \bar{\tau} \gtrsim 2$. This trend is better observed in Fig. 7(b), where $\eta(t)$ estimated from experimental data is shown. As explained in Sec. III this drift is expected in case of dispersion in the modal time constants $\left\{\tau_{k}\right\}$. Once the exponential parameters $A_{o}$ and $\bar{\tau}$ are known, it is possible to infer the value of $c=\Delta \tau / \bar{\tau}$ by fitting (17) to the experimental data. The results in Fig. 7(b) support the explanation of dispersion in the modal $\left\{\tau_{k}\right\}$, as the time evolution of $\eta(t)$ is accurately predicted from a single parameter, i.e., $c$. The fact that a weaker dispersion is observed at higher frequency makes physical sense, since at $2 \mathrm{GHz}$ a wavelength of $15 \mathrm{~cm}$ is likelier to generate more uniform losses over the RC metallic surface than at lower frequency, where modal distributions may present more diverse dissipation patterns [36]. It can be concluded that an exponential PDP should not be taken for granted, and a nontrivial result in its own right, since it provides insight in the statistics of modal time constants. 


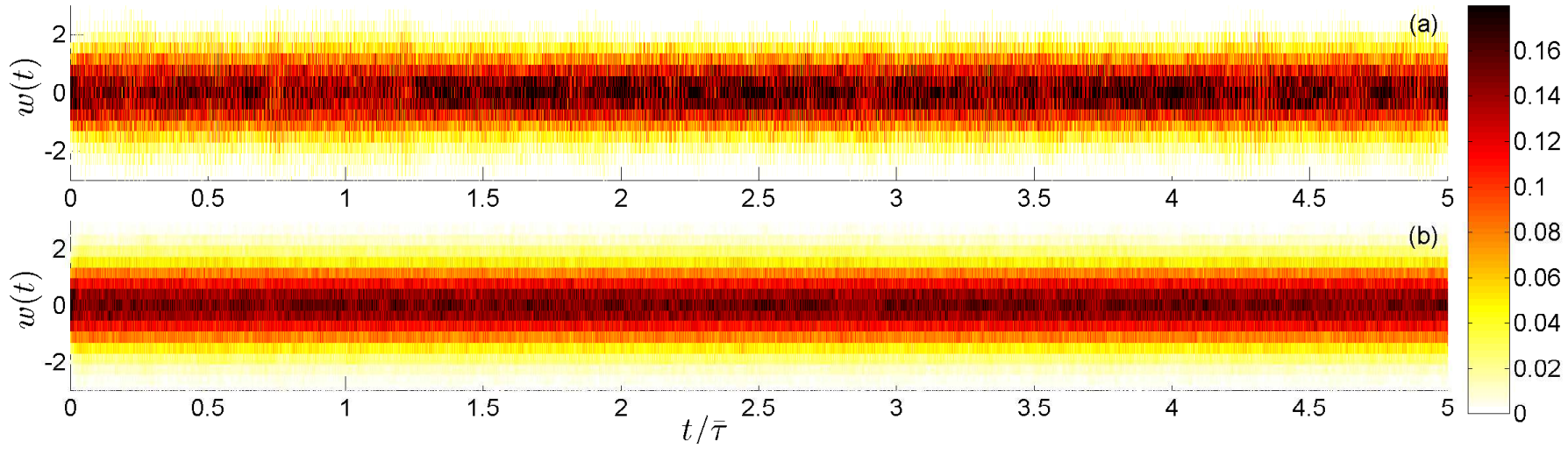

Figure 8: Empirical probability distributions of the process $w(t)$ for $B_{T}=20 \mathrm{MHz}$ and: (a) $f_{c}=0.5 \mathrm{GHz}$ and (b) $f_{c}=2$ GHz. Broad stationarity is observed even for late-time data, to be compared with local deviations highlighted in Fig. 9. The results for $f_{c}=0.5 \mathrm{GHz}$ are more ragged because of the more limited number of realizations available.

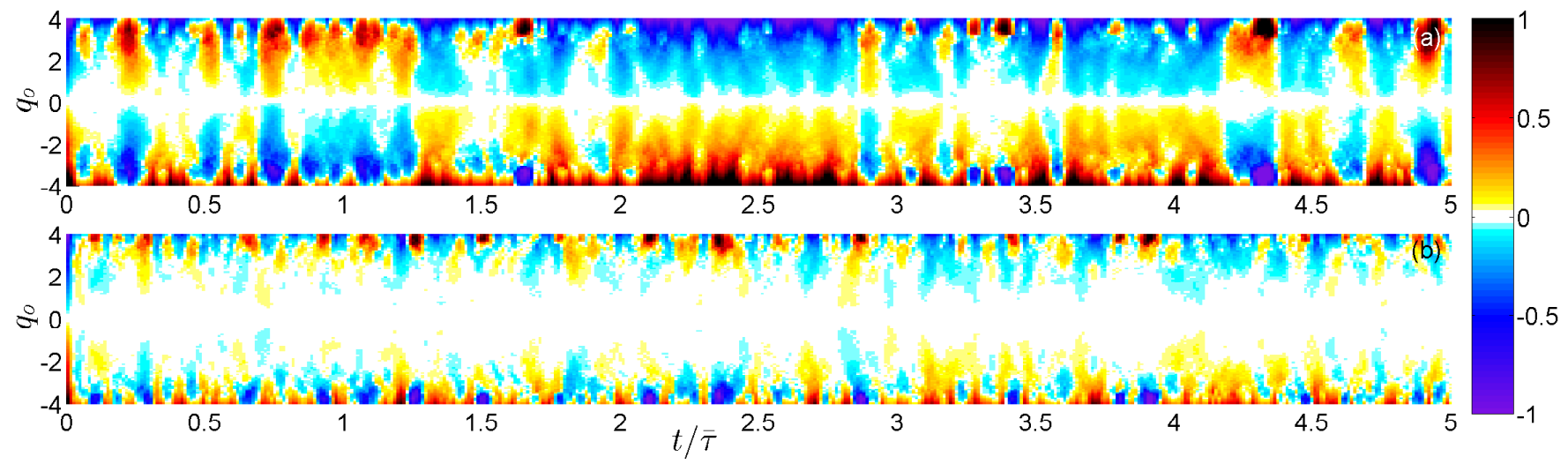

Figure 9: Deviation between empirical and theoretical quantiles $q_{o}$ for the normalized IR $w(t)$, for $B_{T}=20 \mathrm{MHz}$ and: (a) $f_{c}=0.5 \mathrm{GHz}$ and (b) $f_{c}=2 \mathrm{GHz}$. The deviation $q(t)-q_{o}$ is color-coded and represents local departures from Gaussianity.

The realizations of the process $w(t)$ were then computed according to (10), expected from theory to be stationary and following a standard Gaussian probability law. The stationarity can be qualitatively assessed in Fig. 8, where empirical probability distributions, shown as functions of time, do not change significantly in range and amplitude, over the entire time span considered. A steadier behavior was found at $2 \mathrm{GHz}$, whereas at $0.5 \mathrm{GHz}$ the results are less uniform in time. Although the reduced number of samples at $0.5 \mathrm{GHz}$ could be invoked, local variations are more likely due to a limited number of DoF, as discussed below. It should be stressed that if $\eta(t)$ were not taken into account at $0.5 \mathrm{GHz}$, stationarity would not be observed, as the drift from an exponential IR envelope is higher than a factor 2 for $t / \bar{\tau}>5$.

Local drifts from Gaussianity can be assessed quantitatively by computing the quantiles $q(t)$ of the empirical distributions and comparing them with those expected for a standard Gaussian random variable, here named $q_{o}$. The results in Fig. 9 show the absolute error between the two, coded in color, as a function of $q_{o}$ and time. E.g., $q(t)-q_{o}=1$ means that the portion of distribution associated with $q_{o}$ occur further away, i.e., the distribution has heavier tails, while negative deviations imply a contraction. This representation is intrinsically less sensitive to differences in the number of samples, as it is based on cumulative empirical distributions. These results confirm that $w(t)$ at $2 \mathrm{GHz}$ follows more closely a Gaussian distribution for the central quantiles, roughly covering 70 $\%$ of all results (central white region), whereas at $0.5 \mathrm{GHz}$ deviations occur systematically even for the central portion of the distribution.

The hypothesis, formulated in Sec. II, that these deviations are due to a limited number of modes, can be tested experimentally by considering the rms error on the quantiles, computed across time, and correlating it with the number of modes expected for a given frequency and bandwidth. Recalling (3), passing from 0.5 to $2 \mathrm{GHz}$ should lead to a 16-fold increase in the number of DoF, for a fixed $B_{T}$, here set to $15 \mathrm{MHz}$. The same outcome should be observed if $B_{T}$ were increased by a factor 16 while keeping $f_{c}=0.5 \mathrm{GHz}$. Fig. 10 confirms that very similar results are obtained as soon as the same number of DoF are expected, be it for $f_{c}=0.5$ $\mathrm{GHz}$ and $B_{T}=240 \mathrm{MHz}$, or for $f_{c}=2.0 \mathrm{GHz}$ and $B_{T}=15$ MHz. At the same time, a clear trend is visible in Fig. 10, where the rms quantile error steadily decreases as the number of modes increases, as required by the central-limit theorem.

Two reference curves, in thicker solid lines, represent the 

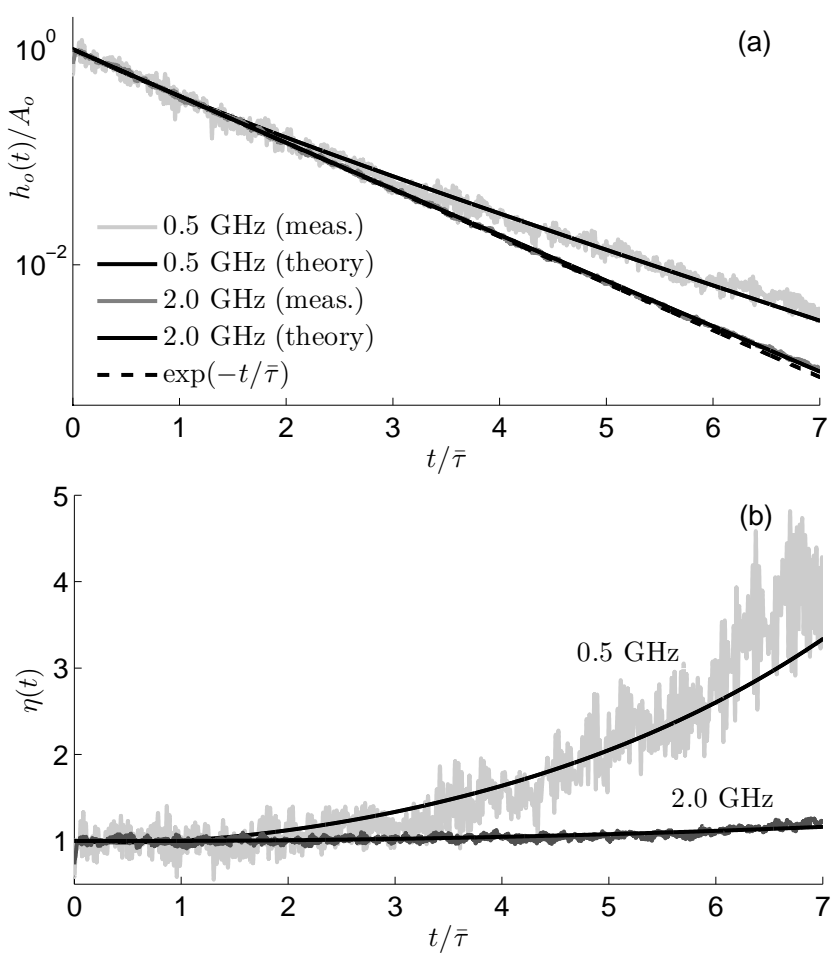

Figure 7: Validation results for the evolution of the IR envelope (i.e., the square root of the PDP) for $B_{T}=20 \mathrm{MHz}$, and $f_{c}=$ 0.5 and $2 \mathrm{GHz}$, comparing experimental results (gray curves) and theory introduced in Sec. III (black curves), for : (a) the envelope and (b) the deviation function $\eta(t, c)$. Optimal fitting of theoretical results to experimental data yield an estimate of $c=0.34$ (for $f_{c}=0.5 \mathrm{GHz}$ ) and $c=0.060$ (for $f_{c}=2$ $\mathrm{GHz}$ ), corresponding to a relative standard deviation of $9.9 \%$ and $1.7 \%$ in the modal time constants $\left\{\tau_{k}\right\}$.

rms error in the empirical quantiles obtained from Monte Carlo simulations involving ideal Gaussian samples, for two populations sizes corresponding to the 0.5 and $2 \mathrm{GHz}$ datasets, respectively. These results are used as references in order to assess whether the finite (and different) size in the 0.5 and $2 \mathrm{GHz}$ datasets could explain the deviations from theoretical Gaussian quantiles. Fig. 10 shows that for the case where a very large number of modes is expected, the quantile rms error is similar to the one found for Gaussian samples, thus indicating convergence. This case occurs for $f_{c}=2 \mathrm{GHz}$ and $B_{T}=240 \mathrm{MHz}$, for a population of 888 samples. On the other hand, as soon as $B_{T}=15 \mathrm{MHz}$, a significant deviation in the empirical quantiles appears, even though the same population size is unchanged. This trend worsens for $f_{c}=0.5 \mathrm{GHz}$, where an even smaller number of modes is expected. These results provide supporting evidence that it is not the size of the datasets that explain the deviations in the quantiles, but rather the changing number of DoF available, here considered to be the resonant modes.

It can be concluded that the predictions of the modal description discussed in Sec. III are robustly supported by experimental data on several grounds. The rms envelope does indeed drift away from the exponential profile usually expected

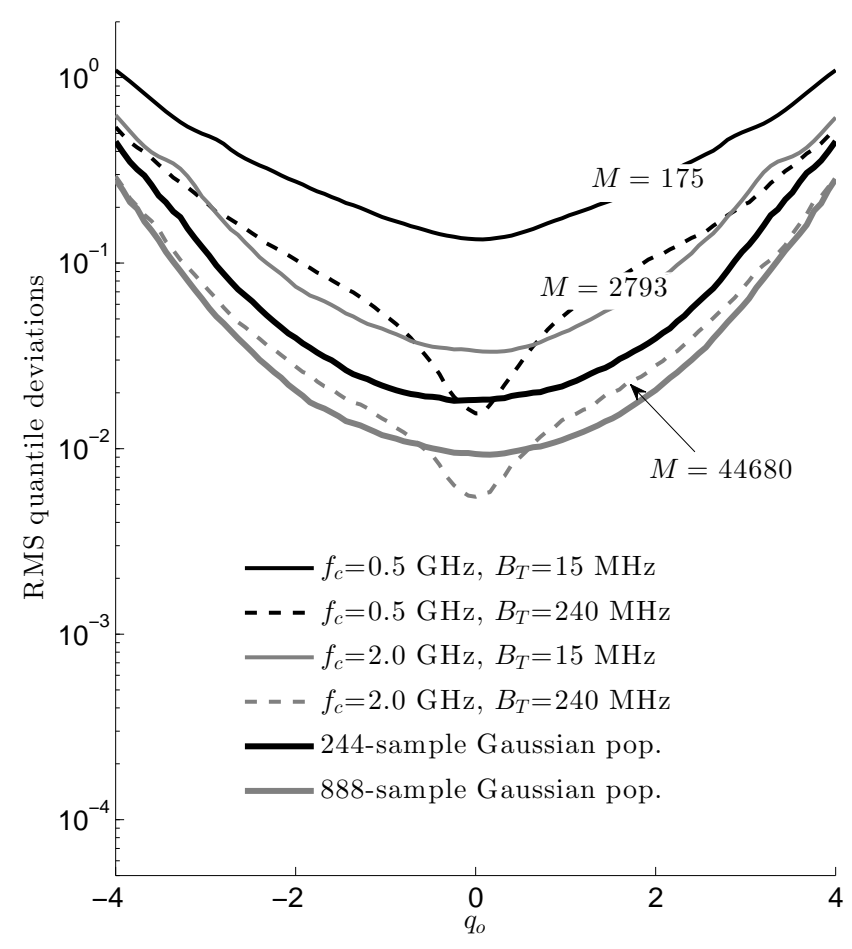

Figure 10: Comparison of the rms deviation between empirical and theoretical quantiles for $w(t)$, as a function of $q_{o}$, for different choices of the excitation bandwidth and frequency. The number of modes available for each configuration is shown close to each curve. The two thicker curves represent the rms quantile deviation expected for ideally Gaussiandistributed populations, of sizes identical to those of the 0.5 $\mathrm{GHz}$ and $2 \mathrm{GHz}$ datasets.

for rich multi-path propagation. The random process $w(t)$ displays a statistical behavior close to a stationary process underlain by a standard Gaussian distribution. The total number $M$ of DoF expected from (3) appear to drive the convergence rate, even when arriving at the same $M$ by choosing two different combinations of frequency and bandwidth. In fact, imperfect convergence to a Gaussian process would hardly be suspected if the number of paths were taken as a measure of the available DoF: at approximatively eight paths per mode, about 1400 paths would be regarded as certain to lead to Rayleigh conditions for $f_{c}=0.5 \mathrm{GHz}$ and $B_{T}=15 \mathrm{MHz}$. Moreover, there is no obvious way of predicting the stronger influence of frequency over $M$ from a multi-path presentation, as opposed to Weyl's formula (3).

\section{Fluctuations from the PDP}

The first fluctuation metric tested dealt with the probability distribution of the overshoot factor $M_{h}$, introduced in Sec. IV, which measures how strongly an IR can exceed the peak of the IR envelope, i.e., $A_{o}$. In case the excitation pulse has a frequency-dependent Fourier spectrum $P(\omega)$, its effects on the coherence time $T_{c}$ must be taken into account by switching from a definition based on the autocorrelation function of 


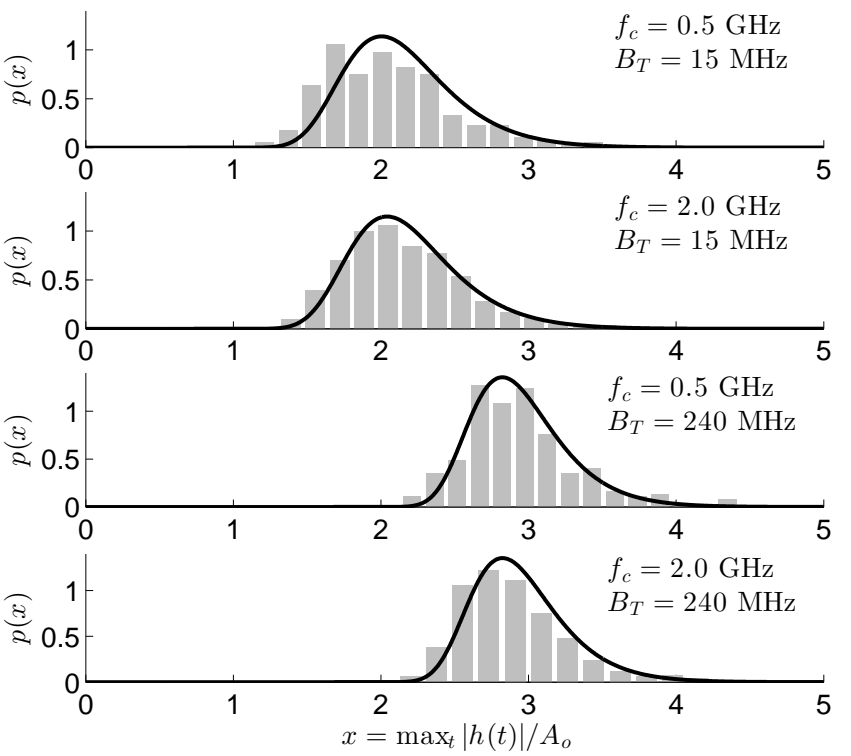

Figure 11: Empirical probability distributions of the peak value observed in IR realizations, for two values of the excitation bandwidth and frequency. Theoretical results predicted by (27) are superposed for validation. Only $B_{T}$ has an impact on the probability distribution, as expected from theory.

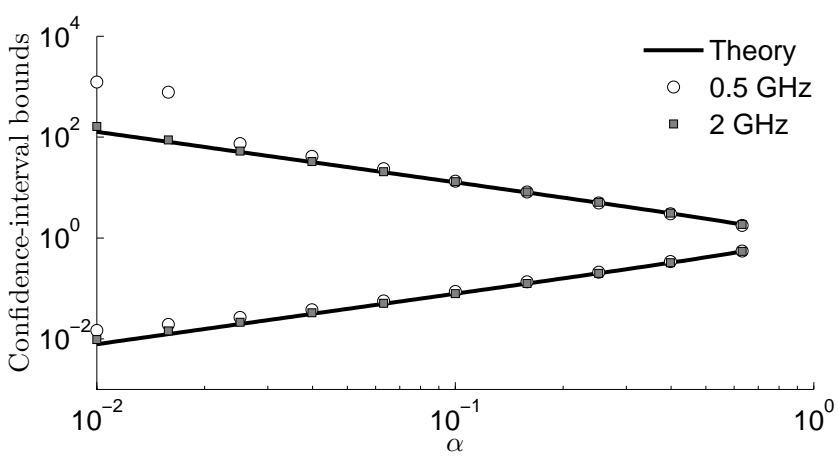

Figure 12: Bounds of the interval of confidence of the dynamic range spanned by $\left|w\left(t+T_{c}\right) / w(t)\right|$, for a varying significance level $\alpha$. Empirical results were obtained for $B_{T}=15 \mathrm{MHz}$ and two central frequencies, spanning all time samples up to $7 \bar{\tau}$.

the IR, to one using the cross-correlation function between the received signal and the test pulse. In this way (38) is generalized as

$$
T_{c}=1 / 4 \kappa^{2} B_{T},
$$

with

$$
\kappa=\left[2 \int \mathrm{d} \omega P(\omega)\right]^{2} / B_{T} \int \mathrm{d} \omega|P(\omega)|^{2},
$$

yielding $\kappa=1.74$ for a Tchebychev window with $-80 \mathrm{~dB}$ side-lobe level, as in (53).

According to theory presented in Sec. IV, only the bandwidth should have an impact on $M_{h}$. Fig. 11 shows the empirical distributions obtained from experimental data, together with the theoretical distribution (29). The results closely match, even though the processes are not perfectly Gaussian (see Sec. VII-B). More ragged results for $f_{c}=0.5 \mathrm{GHz}$ and $B_{T}=15 \mathrm{MHz}$ are observed because of a limited amount of DoF and samples, as already pointed out in Sec. VII-B.

The dynamic range of fluctuations was studied for adjacent samples, for $\Delta t=T_{c}$, as given in (54). Results are shown in Fig. 12, for $B_{T}=15 \mathrm{MHz}$, under the shape of bounds of confidence intervals associated to a given significance probability $\alpha$, which represents the probability of observing samples falling outside the confidence interval. A close agreement between experimental data and (42) is found for $\alpha>2 \%$, with larger errors as $\alpha$ decreases, i.e., in the tails of the probability distribution. As expected from (3), at $0.5 \mathrm{GHz}$ Fig. 12 displays larger errors, as the number of DoF hits a minimum. The expectation that the probability of observing a given ratio $C$ of samples is equal to that for $1 / C$ is confirmed in these results, justifying the strong fluctuations observed in Fig. 1.

The likelihood of recording weak contributions at a time $t$ was assessed in Fig. 13, only for data around $0.5 \mathrm{GHz}$, where deviation from Gaussianity is more likely. The probability of not detecting contributions is a function of the relative sensitivity $s_{o}$, introduced in Sec. VI, here taking four different values, from -40 to $-10 \mathrm{~dB}$. Fig. 13 compares the empirical probabilities obtained from experimental results and those predicted by (44) which agree over a wide time range. The local deviations observed in Fig. 13(a) for $B_{T}=15 \mathrm{MHz}$ are reminiscent of those found in Fig. 9; passing to $B_{T}=240$ $\mathrm{MHz}$ reduces these phenomena, as convergence to asymptotic behavior is more robust. It can be noticed how taking into account the existence of a drift from a purely exponential decay leads to much more accurate results for $s_{o}<-20 \mathrm{~dB}$.

Another measure of the fact that IRs can be highly fragmented is offered by the metric $L_{N}\left(s_{o}\right)$, defined in (45) as the average fraction of time during which $|h(t)| / A_{o}<s_{o}$. Empirical results in Fig. 14 derived from data at $2 \mathrm{GHz}$ are indistinguishable from those predicted by (46), while at 0.5 $\mathrm{GHz}$ a systematic drift is due to having assumed $\eta(t) \simeq 1$. $L_{N}\left(s_{o}\right)$ takes values typically well above $20 \%$, getting close to $50 \%$ as $s_{o} \rightarrow-10 \mathrm{~dB}$. This large variation suggests that on a local level IRs have a high probability of displaying undetectable contributions, a phenomenon that averages out when observed over time on a larger scale.

The fragmentation of IRs leaves room for expecting an average energy recorded over time that could differ from what expected from their PDP. The metric $L_{E}\left(s_{o}\right)$, which measures a potential average difference, is shown in Fig. 14. $L_{E}\left(s_{o}\right)$ appears to be remarkably stable even when $s_{o} \rightarrow-10$ $\mathrm{dB}$, i.e., when the receiver is only able to reliably record contributions from the early-time response of $h(t)$. In the worst case considered, $L_{E}\left(s_{o}\right)$ is reduced by $4 \%$ with respect to the case of infinite sensitivity.

These results confirm that IRs must come as bursts of energy spaced by very weak contributions, as dictated by nonnegligible values of $L_{N}\left(s_{O}\right)$. Since these bursts compensate for the energy that would be expected to be smoothly spread as according to the PDP, their instantaneous power must exceed the one expected for the PDP, consistently with the prediction of an overshoot factor $M_{h}>1$, as well as with an expected 

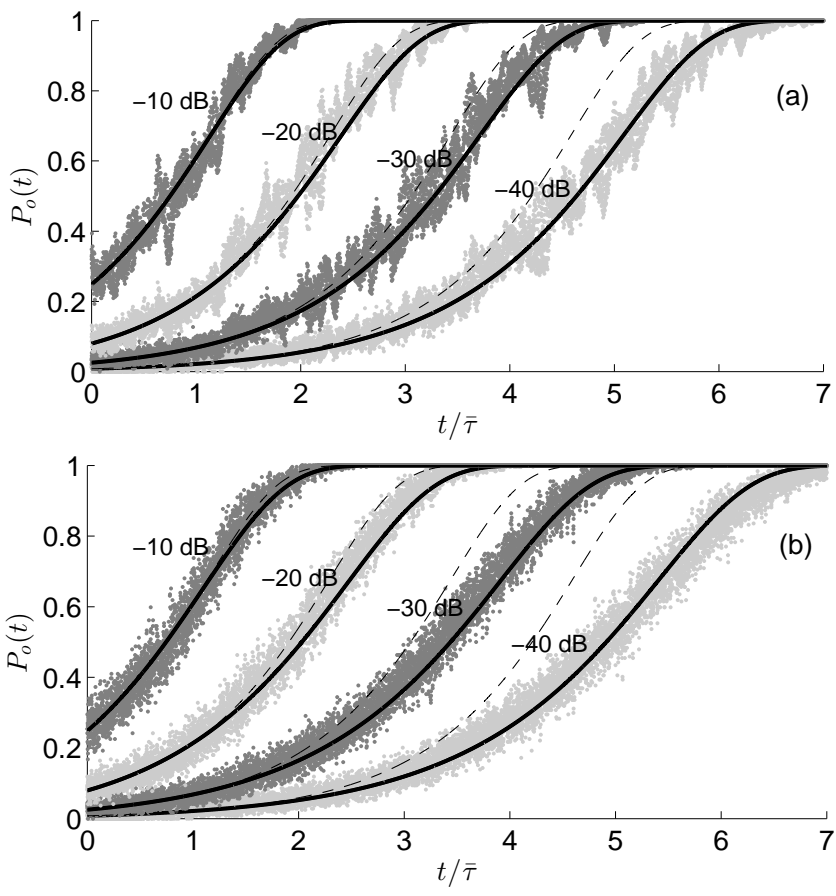

Figure 13: Empirical probability of receiving undetectable contributions, for four relative detection thresholds $s_{o}$, observed at $0.5 \mathrm{GHz}$, for : (a) $B_{T}=15 \mathrm{MHz}$ and (b) $B_{T}=240 \mathrm{MHz}$. Local deviations in (a) are due to the limited number of DoF available and are consistent with those presented in Fig. 9. Theoretical predictions for a purely exponential IR envelope are shown as thin dashed curves, while solid thick curves take into account the correction $\eta(t, c)$ introduced in (17).

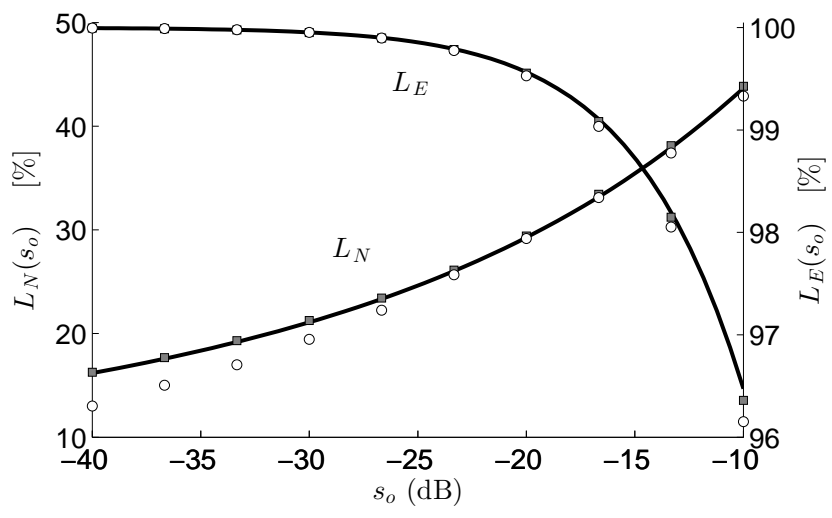

Figure 14: Comparison between empirical and theoretical results for the average fraction of undetected contributions $L_{N}\left(s_{o}\right)$ and the average fraction of detected energy $L_{E}\left(s_{o}\right)$, for $B_{T}=15 \mathrm{MHz}$. Results for 0.5 and $2 \mathrm{GHz}$ are shown using the same symbols as in Fig. 12.

dynamical range where strong and weak contributions occur with the same probability.

\section{CONCLUSIONS}

IRs have been modeled as stochastic processes, starting from a physical description, based on a modal expansion.
A modal approach suggests that the actual number of DoF is given by the number of resonant modes available, which is expected to be highly frequency dependent, a non-trivial prediction when adopting multi-path models. Experimental results confirmed that convergence to a Rayleigh propagation model accelerates at higher frequencies, and is faster than by increasing the bandwidth involved.

The prediction that the IR envelopes (and their PDPs) are only approximatively exponential was also confirmed. Taking these deviations into account, envelope-normalized IRs were confirmed to behave as stationary random processes, even in their late-time response. Local discrepancies from Gaussian processes have been highlighted, and confirmed to reduce as the number of resonant modes increases.

Metrics have been introduced to model the fluctuations of IRs from their envelope. Models derived showed that IRs can locally exceed their rms envelope by a factor easily larger than three, whose evolution is roughly bound by $\ln B_{T} \bar{\tau}$, with $\bar{\tau}$ the average time constant of the medium response. The dynamic range, probability of missing contributions for finite-sensitivity receivers and average detected energy were also studied and fully validated against experimental results.

These results provide insights into the way IRs are controlled by a few physical parameters in a reverberant medium, and are expected to increase awareness about the extents of randomness in IRs. Future work will test these predictions with data collected in real-life reverberant environments, such as indoor structures. Ideally, it would be interesting to use results from datasets described in published papers and compared them in a meta-analysis approach.

\section{REFERENCES}

[1] R. Vaughan and J. B. Andersen, Channels, propagation and antennas for mobile communications. Institution of Electrical Engineers, 2003.

[2] T. K. Sarkar, Z. Ji, K. Kim, A. Medouri, and M. Salazar-Palma, "A survey of various propagation models for mobile communication," Antennas and Propagation Magazine, IEEE, vol. 45, no. 3, pp. 51-82, 2003.

[3] G. Steinböck, M. Gan, P. Meissner, E. Leitinger, K. Witrisal, T. Zemen, and T. Pedersen, "Hybrid model for reverberant indoor radio channels using rays and graphs," IEEE Trans. Antennas Propag., vol. 64, no. 9, pp. 4036-4048, Sept 2016.

[4] A. A. M. Saleh and R. Valenzuela, "A statistical model for indoor multipath propagation," IEEE Journal on Selected Areas in Communications, vol. 5, no. 2, pp. 128-137, February 1987.

[5] D. Cassioli, M. Z. Win, and A. F. Molisch, "The ultra-wide bandwidth indoor channel: from statistical model to simulations," IEEE Journal on Selected Areas in Communications, vol. 20, no. 6, pp. 1247-1257, Aug 2002.

[6] K. Yu, M. Bengtsson, B. Ottersten, D. McNamara, P. Karlsson, and M. Beach, "Modeling of wide-band MIMO radio channels based on NLoS indoor measurements," IEEE Transactions on Vehicular Technology, vol. 53, no. 3, pp. 655-665, May 2004.

[7] V. Erceg, D. G. Michelson, S. S. Ghassemzadeh, L. J. Greenstein, A. J. Rustako, P. B. Guerlain, M. K. Dennison, R. S. Roman, D. J. Barnickel, S. C. Wang, and R. R. Miller, "A model for the multipath delay profile of fixed wireless channels," IEEE Journal on Selected Areas in Communications, vol. 17, no. 3, pp. 399-410, Mar 1999.

[8] P. Yegani and C. D. McGillem, "A statistical model for the factory radio channel," IEEE Transactions on Communications, vol. 39, no. 10, pp. 1445-1454, Oct 1991.

[9] C. L. Holloway, M. G. Cotton, and P. McKenna, "A model for predicting the power delay profile characteristics inside a room," IEEE Trans. Veh. Technol., vol. 48, no. 4, pp. 1110-1120, Jul 1999. 
[10] G. Steinböck, T. Pedersen, B. H. Fleury, W. Wang, and R. Raulefs, "Experimental validation of the reverberation effect in room electromagnetics," IEEE Trans. Antennas Propag., vol. 63, no. 5, pp. 2041-2053, May 2015.

[11] K. Witrisal and M. Pausini, "Statistical analysis of UWB channel correlation functions," IEEE Transactions on Vehicular Technology, vol. 57, no. 3, pp. 1359-1373, May 2008.

[12] S. Chiu, J. Chuang, and D. G. Michelson, "Characterization of UWB channel impulse responses within the passenger cabin of a boeing 737200 aircraft," IEEE Trans. Antennas Propag., vol. 58, no. 3, pp. 935-945, March 2010.

[13] J. B. Andersen, K. L. Chee, M. Jacob, G. F. Pedersen, and T. Kürner, "Reverberation and absorption in an aircraft cabin with the impact of passengers," IEEE Transactions on Antennas and Propagation, vol. 60, no. 5, pp. 2472-2480, 2012.

[14] O. Delangre, S. V. Roy, P. D. Doncker, M. Lienard, and P. Degauque, "Modeling in-vehicle wideband wireless channels using reverberation chamber theory," in 2007 IEEE 66th Vehicular Technology Conference, Sept 2007, pp. 2149-2153.

[15] S. Herbert, T. H. Loh, and I. Wassell, "An impulse response model and Q factor estimation for vehicle cavities," IEEE Transactions on Vehicular Technology, vol. 62, no. 9, pp. 4240-4250, Nov 2013.

[16] E. Genender, C. Holloway, K. Remley, J. Ladbury, G. Koepke, and H. Garbe, "Simulating the multipath channel with a reverberation chamber: Application to bit error rate measurements," Electromagnetic Compatibility, IEEE Transactions on, vol. 52, no. 4, pp. 766-777, Nov 2010.

[17] C. Holloway, D. Hill, J. Ladbury, P. Wilson, G. Koepke, and J. Coder, "On the use of reverberation chambers to simulate a rician radio environment for the testing of wireless devices," Antennas and Propagation, IEEE Transactions on, vol. 54, no. 11, pp. 3167-3177, Nov 2006.

[18] S. Herbert, T. Loh, I. Wassell, and J. Rigelsford, "On the analogy between vehicle and vehicle-like cavities with reverberation chambers," IEEE Transactions on Antennas and Propagation, vol. 62, no. 12, pp. 6236-6245, Dec 2014.

[19] J. Karedal, S. Wyne, P. Almers, F. Tufvesson, and A. Molisch, "Statistical analysis of the UWB channel in an industrial environment," in Vehicular Technology Conference, 2004. VTC2004-Fall. 2004 IEEE 60th, vol. 1, Sept 2004, pp. 81-85 Vol. 1.

[20] P. V. Nikitin, D. D. Stancil, O. K. Tonguz, A. E. Xhafa, A. G. Cepni, and D. Brodtkorb, "Impulse response of the HVAC duct as a communication channel," IEEE Transactions on Communications, vol. 51, no. 10, pp. 1736-1742, Oct 2003.

[21] G. L. Turin, F. D. Clapp, T. L. Johnston, S. B. Fine, and D. Lavry, "A statistical model of urban multipath propagation," IEEE Trans. Veh. Technol., vol. 21, no. 1, pp. 1-9, Feb 1972.

[22] J. A. Dabin, A. M. Haimovich, and H. Grebel, "A statistical ultrawideband indoor channel model and the effects of antenna directivity on path loss and multipath propagation," IEEE Journal on Selected Areas in Communications, vol. 24, no. 4, pp. 752-758, April 2006.

[23] A. F. Molisch, K. Balakrishnan, C.-C. Chong, S. Emami, A. Fort, J. Karedal, J. Kunisch, H. Schantz, U. Schuster, and K. Siwiak, "IEEE 802.15. 4a channel model-final report," IEEE P802, vol. 15, no. 04, p. 0662, 2004.

[24] Q. H. Spencer, B. D. Jeffs, M. A. Jensen, and A. L. Swindlehurst, "Modeling the statistical time and angle of arrival characteristics of an indoor multipath channel," IEEE Journal on Selected Areas in Communications, vol. 18, no. 3, pp. 347-360, March 2000.

[25] S. Y. Tan and H. S. Tan, "Modelling and measurements of channel impulse response for an indoor wireless communication system," IEE Proceedings - Microwaves, Antennas and Propagation, vol. 142, no. 5, pp. 405-, Oct 1995.

[26] H. Kuttruff, Room acoustics. Taylor \& Francis, 2000.

[27] H.-J. Stöckmann, Quantum chaos: an introduction. Cambridge university press, 2006.

[28] B. Liu, D. Chang, and M. Ma, "Eigenmodes and the Composite Quality Factor of a Reverberation Chamber," National Bureau of Standards, Tech. Rep. 1066, 1983.

[29] A. Cozza, "The role of losses in the definition of the overmoded condition for reverberation chambers and their statistics," IEEE Trans. Electromagn. Compat., vol. 53, no. 2, pp. 296-307, May 2011.

[30] F. Monsef and A. Cozza, "Average number of significant modes excited in a mode-stirred reverberation chamber," IEEE Trans. Electromagn. Compat., vol. 56, no. 2, pp. 259-265, 2014.

[31] P. Morse and H. Feshbach, Methods of Theoretical Physics. Feshback Publishing, 1981.

[32] J. Van Bladel, Electromagnetic fields. IEEE, 2007.
[33] A. Cozza, "Source correlation in randomly excited complex media," Antennas and Wireless Propagation Letters, IEEE, vol. 11, pp. 105$108,2012$.

[34] T. Lehman, "A statistical theory of electromagnetic fields in complex cavities," Interaction Notes, Note 494, 1993.

[35] D. Hill, "Electromagnetic Theory of Reverberation Chambers," National Institute of Standards and Technology, Tech. Rep. 1506, 1998.

[36] L. R. Arnaut and G. Gradoni, "Probability distribution of the quality factor of a mode-stirred reverberation chamber," IEEE Trans. Electromagn. Compat., vol. 55, no. 1, pp. 35-44, Feb 2013.

[37] L. Arnaut, P. Besnier, J. Sol, and M. Andries, "On the uncertainty quantification of the quality factor of reverberation chambers," IEEE Trans. Electromagn. Compat., vol. 99, 2018.

[38] H. Nikookar and H. Hashemi, "Phase modeling of indoor radio propagation channels," IEEE Transactions on Vehicular Technology, vol. 49, no. 2, pp. 594-606, Mar 2000.

[39] J. Chakarothai, J. Wang, O. Fujiwara, K. Wake, and S. Watanabe, "Dosimetry of a reverberation chamber for whole-body exposure of small animals," IEEE Transactions on Microwave Theory and Techniques, vol. 61, no. 9, pp. 3435-3445, 2013.

[40] L. De Haan and A. Ferreira, Extreme value theory: an introduction. Springer Science \& Business Media, 2007.

[41] P.-S. Kildal and K. Rosengren, "Correlation and capacity of MIMO systems and mutual coupling, radiation efficiency, and diversity gain of their antennas: simulations and measurements in a reverberation chamber," IEEE Communications Magazine, vol. 42, no. 12, pp. 104112,2004

[42] A. Cozza, F. Masciovecchio, C. Dorgan, M. Serhir, F. Monsef, and D. Lecointe, "A dielectric low-perturbation field scanner for multipath environments," IEEE Transactions on Antennas and Propagation, vol. 65, no. 4, pp. 1978-1987, April 2017.

[43] D. Hill and J. Ladbury, "Spatial-correlation functions of fields and energy density in a reverberation chamber," IEEE Trans. Electromagn. Compat., vol. 44, no. 1, pp. 95-101, 2002.

[44] R. G. Lyons, Understanding digital signal processing. Prentice Hall, 2011.

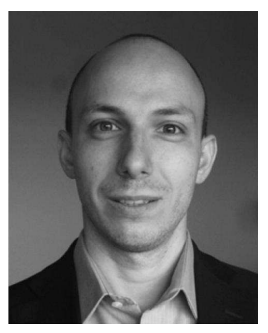

Andrea Cozza (S'02 - M'05 - SM'12) received the Laurea degree (summa cum laude) in electronic engineering from Politecnico di Torino, Turin, Italy, in 2001, and the Ph.D. degree in electronic engineering jointly from Politecnico di Torino and the University of Lille, France, in 2005.

In 2007, he joined the Département de Recherche en Électromagnétisme, SUPELEC (now CentraleSupélec), Gif sur Yvette, France, where since 2013 he is full professor. He is a reviewer for several scientific journals, including those of IET and IEEE. His current research interests include reverberation chambers, statistical electromagnetics, wave propagation through complex media and applications of time reversal to electromagnetics.

Dr. Cozza was awarded the 2012 Prix Coron-Thévenet from the Académie des Sciences, France. 